\title{
Effects of dihydroartemisinin on the gut microbiome of mice
}

\author{
YANYAN LIU ${ }^{1,2^{*}}$, YANHONG YANG $^{3 *}$, YUTING LEI $^{1}$, \\ LANXIANG YANG $^{1,4}$, XUEYING ZHANG ${ }^{1,4}$, JIAN YUAN ${ }^{2}$ and ZILI LEI ${ }^{1}$ \\ ${ }^{1}$ Guangdong Metabolic Disease Research Center of Integrated Chinese and Western Medicine, Institute of Chinese \\ Medicinal Sciences; ${ }^{2}$ Department of Pathology and Guangdong Key Laboratory for Bioactive Drugs Research, \\ Guangdong Pharmaceutical University, Guangzhou Higher Education Mega Center, Guangzhou, Guangdong 510006; \\ ${ }^{3}$ The First Affiliated Hospital (School of Clinical Medicine), Guangdong Pharmaceutical University, Guangzhou, \\ Guangdong 510080; ${ }^{4}$ School of Traditional Chinese Medicine, Guangdong Pharmaceutical University, \\ Guangzhou Higher Education Mega Center, Guangzhou, Guangdong 510006, P.R. China
}

Received September 5, 2019; Accepted April 8, 2020

DOI: $10.3892 / \mathrm{mmr} .2020 .11165$

\begin{abstract}
Dihydroartemisinin (DHA) is a semisynthetic derivative of artemisinin, which has been found to exhibit a broad range of biological activities, excluding antimalarial effects; however its effects on the gut microbiota remain poorly understood. The present study aimed to investigate the effects of DHA on the gut microbiome in mice and to determine its potential biological and pharmaceutical activities through its alteration of the gut microbiota. Serum glucose, triglyceride (TG), total cholesterol, lipopolysaccharide, high density lipoprotein-cholesterol, low density lipoprotein-cholesterol, alanine aminotransferase and aspartate aminotransferase levels in mice treated with DHA were analyzed using the corresponding detection kits. In addition, hematoxylin and eosin staining was performed to determine the pathological effects of DHA on the liver, kidney and intestinal tissues of mice, and the effects of DHA on the gut microbiome were analyzed using 16S ribosomal (r)DNA gene analysis. The results demonstrated that the TG serum levels of mice
\end{abstract}

Correspondence to: Professor Jian Yuan, Department of Pathology and Guangdong Key Laboratory for Bioactive Drugs Research, Guangdong Pharmaceutical University, Guangzhou Higher Education Mega Center, 280 Waihuan East Road, Guangzhou, Guangdong 510006, P.R. China

E-mail: janeyuan9909@163.com

Dr Zili Lei, Guangdong Metabolic Disease Research Center of Integrated Chinese and Western Medicine, Institute of Chinese Medicinal Sciences, Guangdong Pharmaceutical University, Guangzhou Higher Education Mega Center, 280 Waihuan East Road, Guangzhou, Guangdong 510006, P.R. China

E-mail: 3182683090@qq.com

*Contributed equally

Key words: dihydroartemisinin, lipid metabolism, triglyceride, gut microbiome, $16 \mathrm{~S}$ ribosomal DNA gene analysis treated with DHA were significantly decreased compared with the control group. Furthermore, 16S rDNA gene analysis demonstrated that the bacterial diversity of mice treated with DHA was enriched compared with the control group. The DHA group exhibited increased numbers of Firmicutes and Saccharibacteria, and decreased Deferribacteres and Actinobacteria compared with the control group at the phylum level. Kyoto Encyclopedia of Genes and Genomes signaling pathway enrichment analysis also revealed that the signaling pathways associated with 'Energy metabolism' and 'Nucleotide metabolism' were upregulated, whereas the signaling pathways associated with 'Infectious diseases and 'Neurodegenerative diseases' were downregulated in the DHA group compared with the control group. In conclusion, the findings of the present study indicated that DHA may significantly decrease the serum TG levels and alter the gut microbiota, which suggested its potential to be used for the treatment of hyperlipidemia, inflammatory and neurodegenerative disorders.

\section{Introduction}

Artemisinin is a sesquiterpene lactone that is isolated from the Chinese plant Artemisia annua (Qinghao) (1). Artemisinin has been reported to possess antimalarial properties and it has also been discovered to serve roles in several types of disease, including different types of cancer and inflammatory disorders (1-3). Dihydroartemisinin (DHA) is a semisynthetic derivative of artemisinin that has exhibited a broad range of biological activities, excluding antimalarial effects (2). For example, Chen et al (4) demonstrated that DHA ameliorated liver damage, steatosis and hepatocyte lipoapoptosis of alcoholic fatty liver model mice by regulating lipin-1 signaling. Li et al (5) revealed that DHA also inhibited the proliferation and migration of breast cancer cell lines. DHA was also reported to serve important roles in downregulating the prostaglandin E2 synthesis signaling cascade and the inflammation of endothelial cells in the cardiovascular system (6). Furthermore, Zhu and Ji (7) suggested that DHA may have the potential to be used as a treatment option for asthma by 
regulating the microRNA-183C and interleukin-6/STAT3 signaling pathway.

In more recent years, gut microbiota has become a research hotspot due to its involvement in several types of disease, including obesity, diabetes, stroke, allergic asthma, nonalcoholic fatty liver disease/nonalcoholic steatohepatitis, cancer, inflammation, and behavioral and physiological abnormalities (8-10). DHA has demonstrated the potential to be used as a treatment option for fatty liver disease, inflammatory disorders and different types of cancer (4-6), except for malaria; however, its underlying molecular mechanism of action remains unknown. Chen et al (11), reported that artesunate (AS) exhibited the potential to regulate the gut microbiota, ameliorate inflammation and improve the conditions of the liver and intestine following the damage caused by carbon tetrachloride, alcohol and a high fat diet in Sprague Dawley rats, suggesting that DHA may have similar effects on the gut microbiota. Thus, the present study aimed to investigate the effects of DHA in the liver, kidney, intestine and gut microbiota to identify the underlying molecular mechanisms of DHA and its pharmacological activities.

\section{Materials and methods}

Animal studies and tissue collection. The experimental procedures in the present study were approved by the Committee on Laboratory Animal Care and Use of Guangdong Pharmaceutical University (Guangzhou, China). A total of 18 male C57BL/6 mice (weight, 22.53 $1.23 \mathrm{~g}$; age, 7 weeks old; provided by Hunan Lex Jingda Laboratory Animal Co., Ltd.) were housed in a temperature-controlled environment at $25^{\circ} \mathrm{C}$ and $50 \pm 10 \%$ humidity, with a 12 -h light/dark cycle and free access to a standard diet and water. The mice were randomly divided into two groups ( $\mathrm{n}=9 /$ group): Control and DHA group. According to the results obtained in a pre-experiment and previous studies (12-14), the experimental group was treated with DHA $(150 \mathrm{mg} / \mathrm{kg}$; Shanghai Aladdin Biochemical Technology Co., Ltd.) diluted in $0.5 \%$ carboxymethyl cellulose-Na (CMC-Na; Tianjin Zhiyuan Chemical Reagent Co. Ltd.), once daily for 7 days by oral gavage. The control group was treated with $0.5 \% \mathrm{CMC}-\mathrm{Na}$ once daily for 7 days by oral gavage. After 7 days, the whole blood was collected from the orbit; the mice were adequately anesthetized with ether for blood collection and the pinch reflex was monitored to ensure full anesthesia. After $1 \mathrm{ml}$ blood collection, the mice were sacrificed by cervical dislocation. The liver, kidney and intestine tissues were collected, and fixed in $4 \%$ paraformaldehyde overnight at $4^{\circ} \mathrm{C}$ for hematoxylin and eosin (H\&E) staining. The experimental procedures were performed as previously described (15).

Serum lipid profile assays. Serum was obtained from the blood by centrifugation $(1,160 \mathrm{x} \mathrm{g})$ at $4^{\circ} \mathrm{C}$ for $15 \mathrm{~min}$. The concentrations of serum triglyceride (TG), total cholesterol (TC), high density lipoprotein-cholesterol (HDL-C), low density lipoprotein-cholesterol (LDL-C), alanine aminotransferase (ALT) and aspartate aminotransferase (AST) were analyzed using their respective kits (Nanjing Jiancheng Bioengineering Institute; cat. no. A110-1-1 for TG; cat. no. A111-1-1 for TC; cat. no. A112-1-1 for HDL-C; cat. no. A113-1-1 for LDL-C; cat. no. C009-2-1 for ALT; cat. no. C010-2-1 for AST), according to the manufacturer's protocols. The serum glucose levels were detected using a detection kit purchased from Shanghai Rongsheng Biopharmaceutical Co., Ltd., according to the manufacturer's protocols, and the serum liposaccharide (LPS) concentration was determined using a detection kit purchased from Jiangsu Enzyme Immunity Co., Ltd., according to the manufacturer's protocols.

$16 S$ ribosomal (r)DNA gene analysis. Fecal bacterial DNA extraction, 16S rDNA gene amplification and subsequent $16 \mathrm{~S}$ rDNA gene analysis were performed by Gene Denovo Biotechnology Company (Guangzhou, China). The experimental procedures were performed as previously described (15,16). All $\alpha$ diversity indexes were calculated in QIIME (17) (version 1.9.1; http://qiime.org). Shannon rarefaction curves, Operational taxonomic units (OTU) rarefaction curve and rank abundance curves were plotted in $\mathrm{R}$ project ggplot2 package (18) (v2.2.1).

The abundance of bacteria was calculated using the method of Ace and Chao (19), and the diversity of bacteria was estimated using the method of Shannon and Simpson (20). Principal coordinates analysis (Vegan; v2.5-4; https://cran.r-project.org/web/packages/vegan) was performed in order to present the differences between the gut microbial communities of different groups. The Kyoto Encyclopedia of Genes and Genomes (KEGG) pathway analysis was performed at http://www.kegg.jp/kegg/kegg1.html. Canonical Correspondence Analysis (CCA) was performed using Vegan (21), and the correlation network analysis was performed using Psych (v1.8.4; https://cran.r-project.org/web/packages/psych/index.html).

$H \& E$ staining. Mouse tissues were fixed in $4 \%$ paraformaldehyde overnight at $4^{\circ} \mathrm{C}$, dehydrated with ascending series of alcohol (70\% alcohol for $45 \mathrm{~min}, 75 \%$ alcohol for $45 \mathrm{~min}$, $80 \%$ alcohol for $45 \mathrm{~min}, 85 \%$ alcohol for $45 \mathrm{~min}, 95 \%$ alcohol for $45 \mathrm{~min}$, pure alcohol twice for $1 \mathrm{~h}$ each) and embedded in paraffin. Paraffin-embedded tissues were sliced into $4-\mu \mathrm{m}$ sections and stained with hematoxylin for $3 \mathrm{~min}$ and eosin for $20 \mathrm{sec}$, both at room temperature (Sigma-Aldrich; Merck $\mathrm{KGaA}$ ). The stained sections were visualized using an automated quantitative pathology system (Vutomated Quantitative Pathology Imaging System; PerkinElmer, Inc.; the magnification of kidney and liver was $\mathrm{x} 40$, and the magnification of intestinal tissue was $\mathrm{x} 20$ ).

Statistical analysis. Statistical analysis was performed using SPSS software (v23.0; National Institutes of Health) and the data are presented as the mean \pm standard deviation (SD). Statistical differences among the two groups were determined using an unpaired Student's t-test for equal variances or a U-Mann Whitney test for unequal variances. Each experiment was repeated $\geq 3$ times. $\mathrm{P}<0.05$ was considered to indicate a statistically significant difference.

\section{Results}

Effects of DHA on the serum lipid profile and important organs. DHA was demonstrated to significantly decrease the 

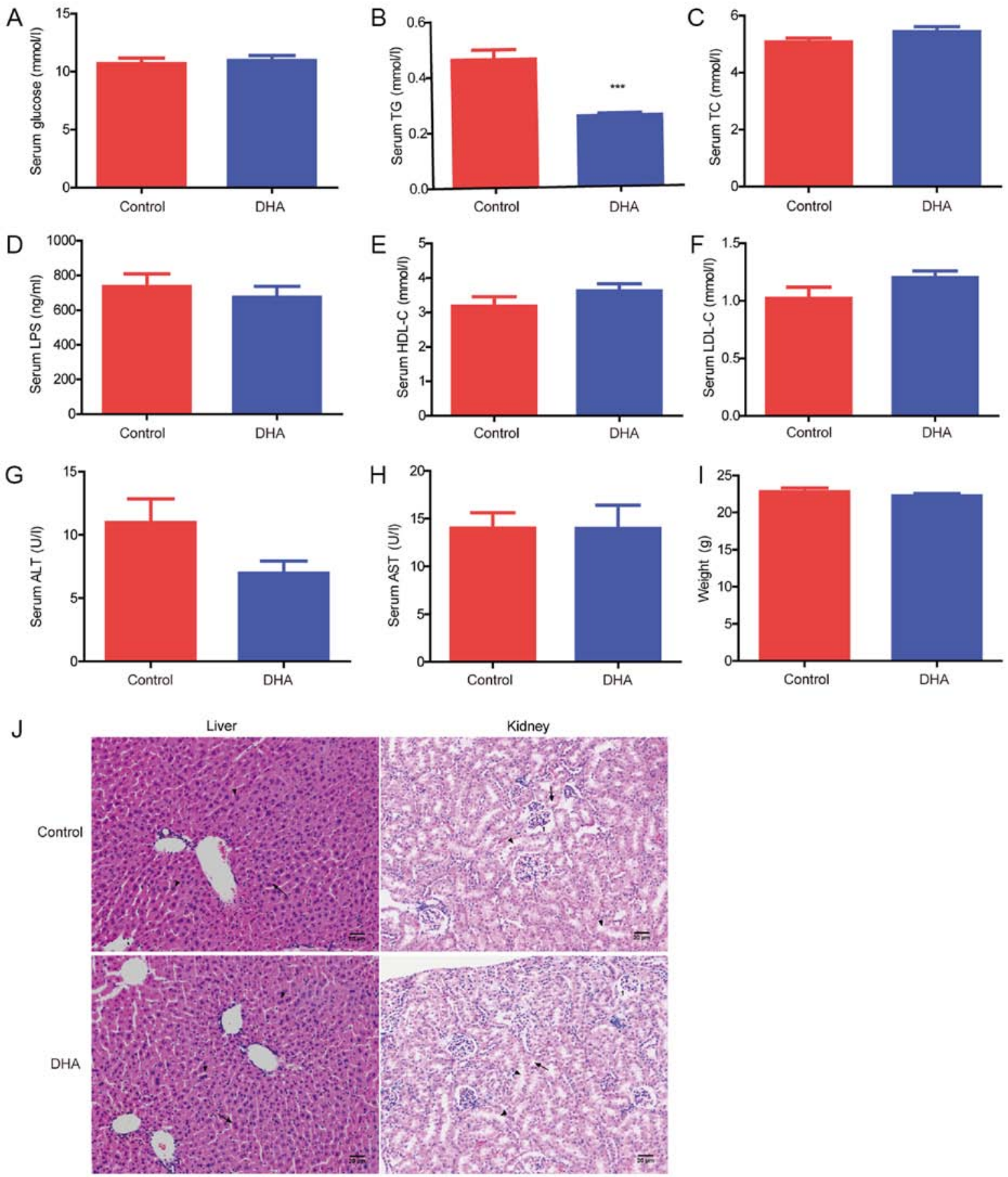

Figure 1. Effects of DHA on the serum lipid profile and important organs. Serum (A) glucose, (B) TG, (C) TC, (D) LPS, (E) HDL-C, (F) LDL-C, (G) ALT and (H) AST levels were analyzed in the DHA and control groups. (I) Body weight of the mice in the DHA and control groups. (J) Hematoxylin and eosin staining of the liver and kidney tissues obtained from the DHA and control groups. Scale bar, $20 \mu \mathrm{m}$. In the liver: Arrows, hepatocytes; triangles, hepatic cords. In the kidney: 1, spherical renal corpuscles; arrows, proximal convoluted tubule; triangles, distal convoluted tubule. ${ }^{* * * *} \mathrm{P}<0.001$ vs. control. TG, triglyceride; TC, total cholesterol; LPS, lipopolysaccharide; HDL-C, high density lipoprotein-cholesterol; LDL-C, low density lipoprotein-cholesterol; ALT, alanine aminotransferase; AST, aspartate aminotransferase; DHA, dihydroartemisinin.

serum TG levels compared with the control group (Fig. 1B); however, no significant differences were observed in the serum glucose, TC, LPS, HDL-C, LDL-C, ALT and AST levels, or the body weight, between the DHA and control groups (Fig. 1A, C-I). H\&E staining revealed that the liver and kidney tissues of the DHA group were similar in pathology compared with the control group (Fig. 1J). Furthermore, no significant differences were observed in the pathology of the intestine, including the duodenum, jejunum, ileum and colon, between the two groups (Fig. S1).

Overview of the $16 S$ rDNA gene sequencing. In order to determine the effect of DHA on the gut microbiota, mice fecal samples were collected and the 16S rDNA genes were sequenced. The indices of Shannon and Simpson (calculated for the diversity of bacteria), Chao and Ace (calculated for 
Table I. Diversity estimation of the $16 \mathrm{~S}$ ribosomal RNA gene library of the DHA-treated mice and the control group.

\begin{tabular}{lcrrrrr}
\hline Group & Total tags & $\begin{array}{c}\text { Operational } \\
\text { taxonomic units }\end{array}$ & Shannon & Simpson & Chao & Ace \\
\hline Control & $119009.11 \pm 17477.13$ & $953.22 \pm 84.17$ & $6.09 \pm 0.12$ & $0.97 \pm 0.01$ & $1661.94 \pm 104.26$ & $1717.02 \pm 110.90$ \\
DHA & $132965.38 \pm 10623.35$ & $1010.13 \pm 51.92$ & $6.32 \pm 0.27$ & $0.97 \pm 0.01$ & $1763.40 \pm 149.16$ & $1728.54 \pm 120.93$ \\
\hline
\end{tabular}

DHA, dihydroartemisinin.

Table II. Taxonomic composition of the gut bacteria on the phylum level of the DHA and control groups.

\begin{tabular}{lrrrr}
\hline Phylum & Control (\%) & DHA (\%) & DHA/control & P-value \\
\hline Bacteroidetes & 63.61675556 & 59.1229500 & 0.92936129 & 0.262040377 \\
Firmicutes & 19.26385556 & 27.8502625 & 1.44572629 & 0.046519675 \\
Verrucomicrobia & 8.32234444 & 7.2688500 & 0.87341374 & 0.723052299 \\
Proteobacteria & 7.55047778 & 5.1879000 & 0.68709559 & 0.119181387 \\
Deferribacteres & 0.67160000 & 0.0777500 & 0.11576831 & 0.002711787 \\
\end{tabular}

the abundance of bacteria), together with the total tags and OTUs of each group are presented in Table I. The Shannon rarefaction curves for each group were observed to reach the saturation plateau, suggesting the sufficient sequence coverage of the samples to describe the composition of the gut microbiota (Fig. 2A). The Venn diagram demonstrated that there were 597 common OTUs identified between the DHA and control groups, and there were 195 and 110 OTUs specific to the DHA-treated mice and the control group, respectively (Fig. 2B).

Effect of DHA on the relative abundance and composition of the gut microbiota of mice. The $\alpha$ diversity analysis of the two groups demonstrated that the bacterial diversity of the DHA group was richer compared with the control group (Fig. 2C). Furthermore, principal coordinates analysis revealed that the DHA and the control groups could be distinguished from each other (Fig. 2D), suggesting that DHA may alter the gut bacteria of the mice.

The taxonomic compositions of the bacterial phylum of the two groups are presented in Fig. 3A and B. The majority of the samples exhibited high percentages of Bacteroidetes, Firmicutes, Verrucomicrobia and Proteobacteria on the phylum level, and the DHA group had increased Firmicutes and Saccharibacteria species, and decreased Deferribacteres and Actinobacteria species compared with the control group (Fig. 3A and B; Table II). The taxonomic compositions of the two groups on the class and family level are presented in Fig. S2, Table SI and Table SII.

The heatmap of KEGG signaling pathway enrichment analysis demonstrated that the following signaling pathways were upregulated in the DHA group: 'ABC transporters', 'Two-component system', 'Purine metabolism', 'Pyrimidine metabolism', 'Amino sugar and nucleotide sugar metabolism', 'Starch and sucrose metabolism', 'Arginine and proline metabolism', 'Peptidoglycan biosynthesis', 'Fructose and mannose metabolism', 'Homologous recombination', 'Nitrogen metabolism', 'Cell cycle-Caulobacter', 'Methane metabolism' and 'Alanine, aspartate and glutamate metabolism' (Fig. 4A). Conversely, the following signaling pathways were downregulated in the DHA group compared with the control group: 'Aminoacyl-tRNA biosynthesis', 'Ribosome', 'Oxidative phosphorylation', 'Bacterial secretion system', 'RNA degradation' and 'Porphyrin and chlorophyll metabolism' (Fig. 4A). The signaling pathways associated with 'Energy Metabolism' and 'Nucleotide Metabolism' were demonstrated to be significantly upregulated in the DHA group compared with the control group (Fig. 4B), while the signaling pathways associated with 'Infectious Diseases' and 'Neurodegenerative Diseases' were significantly downregulated in the DHA group compared with the control group (Fig. 4B). The associations between HDL-C, LDL-C, TG, TC and LPS with the bacterial taxa are presented in Fig. S3. The result of CCA demonstrated a correlation of the bacteria with different environmental factors, and that LDL-C, LPS and TC influenced the structure and composition of gut microbiota most (Fig. S3A). The result of the correlation network analysis indicated that LDL-C had a positive correlation with Verrucomicrobia, but had a negative correlation with Bacteroidetes. HDL had a positive correlation with Saccharibacteria, and TC had a positive correlation with Fimicutes, but had a negative correlation with Proteobacteria. Furthermore, TG had a positive correlation with Deferribacteres, but had a negative correlation with Saccharibacteria (Fig. S3B).

\section{Discussion}

Artemisinin is well-known for its significant role in the treatment of malaria (22) and it was previously demonstrated that artemisinin had the potential to manage 'artemisinin-resistance' (23). In addition to its antimalarial properties, artemisinin and its derivatives were discovered to exhibit 
A

$$
=\underset{\mathrm{DHA}}{\text { Control }}
$$
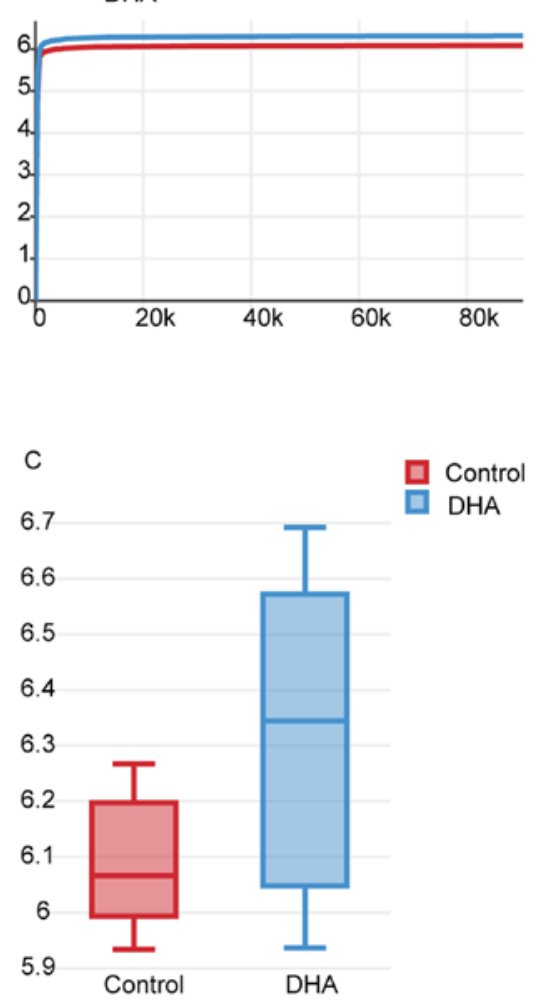

B
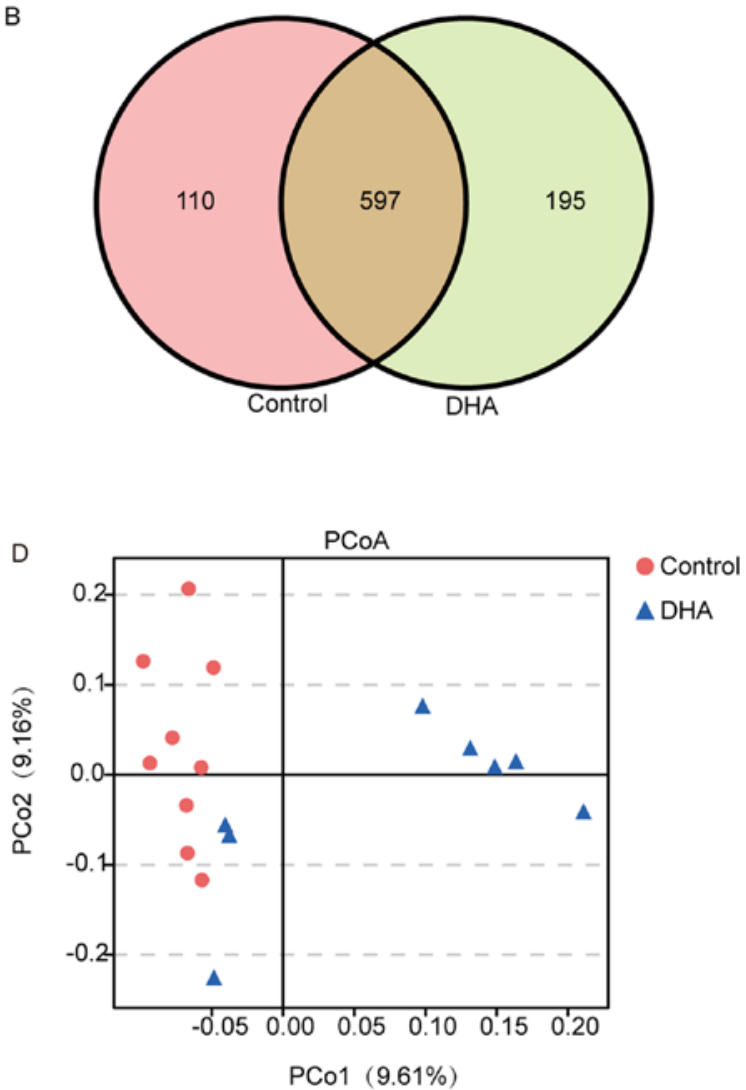

Figure 2. Overview of the $16 \mathrm{~S}$ ribosomal DNA gene analysis. (A) Shannon rarefaction curves for the control and DHA groups. (B) Venn diagram of the DHA and control groups demonstrated the number of OTUs of the control group and the DHA group. The control group had 110 specific OTUs, the DHA group had 195 OTUs and the two groups had 597 OTUs in common. (C) $\alpha$ diversity of the DHA and control groups. (D) PCo analysis of the gut microbiota in the DHA and control groups. DHA, dihydroartemisinin; PCo, Principle coordinates; OTU, Operational taxonomic units.

A

A

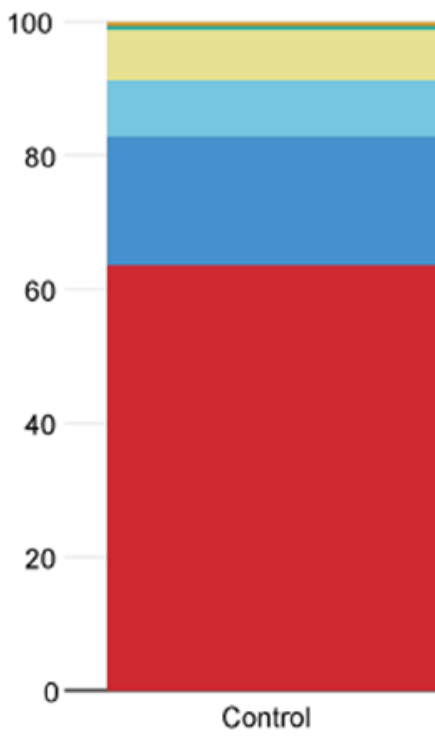

B

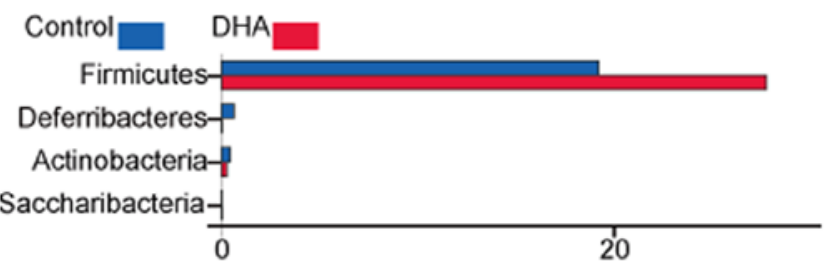

Unclassified

Other

- Fusobacteria

- Tenericutes

Saccharibacteria

Cyanobacteria

Actinobacteria

Deferribacteres

\section{Proteobacteria}

Verrucomicrobia

Frimicutes

Bacteriodetes

Figure 3. Relative abundance of the dominant gut microbiota found in the DHA and control groups. (A and B) Relative abundance of the gut microbiota at the bacterial phylum level in the two groups. DHA, dihydroartemisinin.

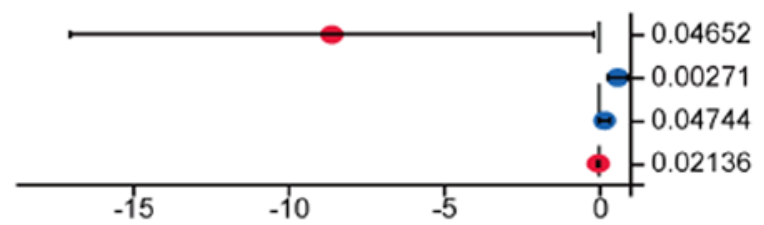


A

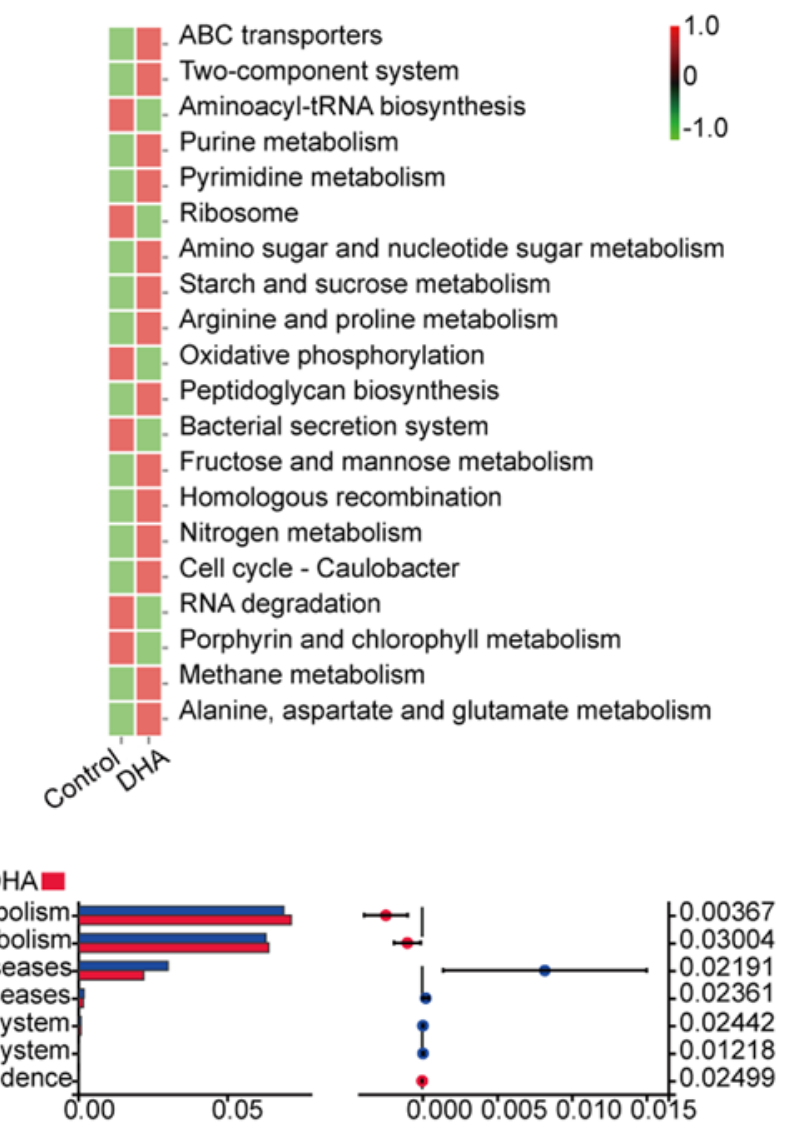

Figure 4. Kyoto Encyclopedia of Genes and Genomes signaling pathway enrichment analysis of the altered signaling pathways in the DHA and control groups. (A) Top 20 signaling pathways in the DHA and control groups. (B) Significantly altered signaling pathways in the DHA and control groups. DHA, dihydroartemisinin.

a range of pharmaceutical activities, such as anti-viruses, anti-inflammation and anti-tumors (1). DHA, an important derivative of artemisinin, was revealed to be associated with several biological activities, including improving liver damage, inhibiting cancer and inflammation (4-7); however, its effect on the gut microbiota remains poorly understood. In the present study, C57BL/6 mice were treated with DHA to determine its effects on the gut microbiota, lipid metabolism and important organs, including the liver, kidney and intestine. The H\&E staining results of the liver, kidney and intestinal tissues demonstrated that there were no significant differences in the pathology between the DHA group and the control group, indicating that DHA did not exert toxic effects on these organs. TG serum levels were significantly decreased in the DHA group compared with the control group; however, there was no significant differences observed in the serum levels of glucose, TC, LPS, HDL-C, LDL-C, ALT and AST between the two groups. It was previously reported that AS significantly decreased the TG plasma levels, thus these findings suggested the potential of DHA to be used as a treatment for hyperlipidemia when combined with ursolic acid $(24,25)$. The results of the present study indicated that DHA may exert similar biological functions as AS.

The effects of DHA on the gut microbiota were further analyzed via $16 \mathrm{~S}$ rDNA gene analysis; the $\alpha$ diversity accounts for the abundance of species residing in the host, whereby a high $\alpha$ diversity indicates a healthy host pattern. For example, Wolff et al (26) reported that the healthy oral microbiome had a significantly higher diversity compared with the advanced caries microbiome, whereas Clarke et al (27) reported that the gut microbiota of athletes was more diverse compared with the controls. The results of the present study demonstrated that the bacterial diversity of the DHA group was richer compared with the control group, suggesting that DHA may have the potential to increase the diversity of the gut bacteria.

Obese individuals have exhibited a tendency to have an increased ratio of Firmicutes to Bacteroidetes in the gut microbiota compared with lean humans and mice (28-30). In the present study, DHA was demonstrated to increase the relative abundance of the Firmicutes phylum from 19.26 to $27.85 \%$, while the Bacteroidetes phylum decreased from 63.62 to $59.12 \%$ compared with the control group, respectively. Although DHA increased the ratio of Firmicutes to Bacteroidetes, the mice treated with DHA failed to exhibit an obese phenotype as there was no difference in body weight between the control and DHA groups. Furthermore, there were no significant differences observed in the body weights of the mice between the DHA and control groups. According to Mazloom et al (31), the importance of the Firmicutes to Bacteroidetes ratio in obesity remains controversial as several studies have reported no significant differences between the two phyla in obesity. The results of the present study suggested that the effect of DHA on the Firmicutes to Bacteroidetes ratio was not associated with obesity. The present study also revealed that DHA significantly decreased TG serum levels, whilst KEGG signaling pathway enrichment analysis indicated that the signaling pathways asso- 
ciated with 'Energy Metabolism' and 'Nucleotide Metabolism', such as 'Purine metabolism', 'Pyrimidine metabolism', 'Amino sugar and nucleotide sugar metabolism', 'Starch and sucrose metabolism', 'Arginine and proline metabolism', 'Peptidoglycan biosynthesis', 'Fructose and mannose metabolism', 'Nitrogen metabolism', 'Methane metabolism' and 'Alanine, aspartate and glutamate metabolism' were upregulated in the DHA group compared with the control group. Overall, these results suggest that DHA may affect lipid metabolism by regulating the gut microbiota. The effects of DHA on the gut microbiota were evaluated in the present study. A high fat diet was previously discovered to affect the relative abundance and composition of the gut microbiota (15). To ensure that the alterations of the gut microbiota were due to the effects of DHA, mice were fed a normal diet in the present study. Since the serum TG levels were observed to be significantly decreased in the DHA group, the effect of DHA on the lipid metabolism will be further evaluated using mice fed with a high fat diet in our future study.

In the present study, KEGG signaling pathway enrichment analysis revealed that the signaling pathways involved in 'Infectious Diseases' and 'Neurodegenerative Diseases' were significantly downregulated in the DHA group compared with the control group. Notably, Liu et al (2) reported that DHA had the potential to suppress the NF- $x \mathrm{~B}$ signaling pathway and inhibit the inflammatory response in lipopolysaccharide-induced septic acute kidney injury within a mouse model. In addition, Kim et al (32) reported that artemisinin inhibited the interaction between nod-like receptor protein 3 (NLRP3) and never in mitosis geneA-related kinase 7 (Nek7) in NLRP3 inflammasome activation. Furthermore, it was indicated that artemisinin may inhibit AMP kinase/NF- $x$ B/NLRP3 inflammasome signaling in macrophages in order to protect the aorta from atherosclerotic lesions (33). Neurodegenerative diseases are characterized by the deterioration of neuronal structures and functions (34), and it has been suggested that artemisinin may be used as a treatment for neurodegenerative diseases by inhibiting oxidation, inflammation and decreasing the level of amyloid $\beta$ protein (34). The results of the present study indicated that DHA may suppress the signaling pathways involved in 'Infectious Diseases' and 'Neurodegenerative Diseases' by regulating the gut microbiota.

In conclusion, the findings of the present study revealed that DHA decreased the TG serum levels in mice and 16S rDNA gene analysis suggested that DHA may have the potential to increase the diversity of the gut bacteria. Mice treated with DHA exhibited increased numbers of Firmicutes and Saccharibacteria, and decreased Deferribacteres and Actinobacteria on the phylum level compared with the control group. KEGG signaling pathway enrichment analysis subsequently demonstrated that DHA upregulated the signaling pathways involved in 'Energy Metabolism' and 'Nucleotide Metabolism', while downregulating the signaling pathways involved in 'Infectious Diseases' and 'Neurodegenerative Diseases'. Altogether, these results suggested that DHA may exert its functions, including decreasing the serum TG levels and inducing anti-inflammatory and anti-neurodegenerative responses through regulating the composition of the gut microbiota.

\section{Acknowledgements}

Not applicable.

\section{Funding}

The present study was supported by the National Natural Science Foundation of China (grant nos. 81803912 and 31671520), the Scientific Research Project of the Administration of Traditional Chinese Medicine of Guangdong Province (grant no. 20182079), the Characteristic Innovation Project (Natural Science) of the Education Department of Guangdong Province, the 'Innovation Strong School Project' of Guangdong Pharmaceutical University (grant no. 2017KTSCX102) and the Science and Technology Project of Yue-Xiu District of Guangzhou (grant no. 2018-WS-011).

\section{Availability of data and materials}

The datasets used and/or analyzed during the current study are available from the corresponding author on reasonable request.

\section{Authors' contributions}

ZL and JY designed and conceived the study; YYL established the model mice and performed the serum analysis; YY designed the study, analyzed and interpreted the results, wrote the first draft of the manuscript and critically revised the manuscript; and YTL, LY and XZ performed the H\&E staining. All authors read and approved the final manuscript.

\section{Ethics approval and consent to participate}

The experimental procedures in the present study were approved by the Committee on Laboratory Animal Care and Use of Guangdong Pharmaceutical University (Guangzhou, China).

\section{Patient consent for publication}

Not applicable.

\section{Competing interests}

The authors declare that they have no competing interests.

\section{References}

1. Ho WE, Peh HY, Chan TK and Wong WS: Artemisinins: Pharmacological actions beyond anti-malarial. Pharmacol Ther 142: 126-139, 2014.

2. Liu X, Lu J, Liao Y, Liu S, Chen Y, He R, Men L, Lu C, Chen Z, Li S, et al: Dihydroartemisinin attenuates lipopolysaccharide-induced acute kidney injury by inhibiting inflammation and oxidative stress. Biomed Pharmacother 117: 109070, 2019.

3. Lu F, He XL, Richard C and Cao J: A brief history of artemisinin: Modes of action and mechanisms of resistance. Chin J Nat Med 17: 331-336, 2019.

4. Chen X, Bian M, Jin H, Lian N, Shao J, Zhang F and Zheng S: Dihydroartemisinin attenuates alcoholic fatty liver through regulation of lipin-1 signaling. IUBMB Life 71: 1740-1750, 2019.

5. Li Y, Zhou X, Liu J, Gao N, Yang R, Wang Q, Ji J, Ma L and He Q: Dihydroartemisinin inhibits the tumorigenesis and metastasis of breast cancer via downregulating CIZ1 expression associated with TGF- $\beta 1$ signaling. Life Sci 248: 117454, 2020.

6. Yin J, Xia W, Zhang Y, Ding G, Chen L, Yang G, Huang S, Jia Z and Zhang A: Role of dihydroartemisinin in regulating prostaglandin E2 synthesis cascade and inflammation in endothelial cells. Heart Vessels 33: 1411-1422, 2018. 
7. Zhu Hand Ji W: Dihydroartemisinin ameliorated ovalbumin-induced asthma in mice via regulation of miR-183C. Med Sci Monit 25: 3804-3814, 2019.

8. Schroeder BO and Bäckhed F: Signals from the gut microbiota to distant organs in physiology and disease. Nat Med 22: 1079-1089, 2016.

9. Kong CY, Li ZM, Han B, Zhang ZY, Chen HL, Zhang SL, Xu JQ, Mao YQ, Zhao YP and Wang LS: Diet consisting of balanced yogurt, fruit and vegetables modifies the gut microbiota and protects mice against nonalcoholic fatty liver disease. Mol Nutr Food Res 63: e1900249, 2019.

10. Kareva I: Metabolism and gut microbiota in cancer immunoediting, CD8/Treg ratios, immune cell homeostasis and implications for cancer (immuno) therapy: Concise Review. Stem Cells 37: 1273-1280, 2019.

11. Chen YX, Lai LN, Zhang HY, Bi YH, Meng L, Li XJ, Tian XX, Wang LM, Fan YM,Zhao ZF, et al: Effect of artesunate supplementation on bacterial translocation and dysbiosis of gut microbiota in rats with liver cirrhosis. World J Gastroenterol 22: 2949-2959, 2016

12. Efferth T: From ancient herb to modern drug: Artemisia annua and artemisinin for cancer therapy. Semin Cancer Biol 46: 65-83, 2017.

13. Olliaro PL, Nair NK, Sathasivam K, Mansor SM and Navaratnam V: Pharmacokinetics of artesunate after single oral administration to rats. BMC Pharmacol 1: 12, 2001.

14. Dai T, Jiang W, Guo Z, Xie Y and Dai R: Comparison of in vitro/in vivo blood distribution and pharmacokinetics of artemisinin, artemether and dihydroartemisinin in rats. J Pharm Biomed Anal 162: 140-148, 2019.

15. Yang Y, Yang F, Huang M, Wu H, Yang C, Zhang X, Yang L, Chen G, Li S, Wang Q, et al: Fatty liver and alteration of the gut microbiome induced by diallyl disulfide. Int J Mol Med 44: 1908-1920, 2019.

16. Yang Y, Lei Z, Huang L and Yang F, Zhang N, Yuan J, Li K, Chen $\mathrm{J}$ and $\mathrm{Zhang} \mathrm{J}$ : Antitumor ability of berberine accompanied by modulation of gut microbiome in sarcoma-180 tumor-bearing mice. Int J Pharmacol 14: 460-470, 2018.

17. Caporaso JG, Kuczynski J, Stombaugh J, Bittinger K, Bushman FD, Costello EK, Fierer N, Peña AG, Goodrich JK, Gordon JI, et al: QIIME allows analysis of high-throughput community sequencing data. Nat Methods 7: 335-336, 2010.

18. Valero-Mora PM: ggplot2: Elegant Graphics for Data Analysis. Journal of Statistical Software 35: 2010.

19. Kemp PF and Aller JY: Bacterial diversity in aquatic and other environments: What $16 \mathrm{~S}$ rDNA libraries can tell us. FEMS Microbiol Ecol 47: 161-177, 2004

20. Frosini BV: Descriptive measures of ecological diversity. In: Environmetrics, in Encyclopedia of Life Support Systems (EOLSS). Jureckova J and El-Shaarawi AH (eds.). Eolss Publishers, Oxoford, UK, 2004.

21. Oksanen J,Blanchet FG, Kindt R,Legendre P,O'Hara RB, Simpson GL, Solymos P, Stevens MHH and Wagner H: Vegan: Community Ecology Package. R package v1.17-12, http://cran.r-project.org/. 2011.
22. Tu Y: Artemisinin-a gift from traditional Chinese medicine to the world (Nobel Lecture). Angew Chem Int Ed Engl 55:10210-10226, 2016.

23. Wang J, Xu C, Liao FL, Jiang T, Krishna S and Tu Y: A Temporizing Solution to "Artemisinin Resistance". N Engl J Med 380: 2087-2089, 2019.

24. Yuliang W, Zejian W, Hanlin S, Ming Y and Kexuan T: The hypolipidemic effect of artesunate and ursolic acid in rats. Pak J Pharm Sci 28: 871-874, 2015

25. Wang YL, Wang ZJ, Shen HL, Yin M and Tang KX: Effects of artesunate and ursolic acid on hyperlipidemia and its complications in rabbit. Eur J Pharm Sci 50: 366-371, 2013.

26. Wolff D, Frese C, Schoilew K, Dalpke A, Wolff B and Boutin S: Amplicon-based microbiome study highlights the loss of diversity and the establishment of a set of species in patients with dentin caries. PLoS One 14: e0219714, 2019.

27. Clarke SF, Murphy EF, O'Sullivan O, Lucey AJ, Humphreys M, Hogan A, Hayes P, O'Reilly M, Jeffery IB, Wood-Martin R, et al: Exercise and associated dietary extremes impact on gut microbial diversity. Gut 63: 1913-1920, 2014.

28. Hills RD Jr, Pontefract BA, Mishcon HR, Black CA, Sutton SC and Theberge CR: Gut microbiome: Profound implications for diet and disease. Nutrients 11: E1613, 2019.

29. Wu R, Zhao D, An R, Wang Z, Li Y, Shi B and Ni Q: Linggui Zhugan Formula improves glucose and lipid levels and alters gut microbiota in high-fat diet-induced diabetic mice. Front Physiol 10: 918, 2019.

30. Song B, Zhong YZ, Zheng CB, Li FN, Duan YH and Deng JP: Propionate alleviates high-fat diet-induced lipid dysmetabolism by modulating gut microbiota in mice. J Appl Microbiol 127: 1546-1555, 2019.

31. Mazloom K, Siddiqi I and Covasa M: Probiotics: How effective are they in the fight against obesity? Nutrients 11: E258, 2019.

32. Kim SK, Choe JY and Park KY: Anti-inflammatory effect of artemisinin on uric acid-induced NLRP3 inflammasome activation through blocking interaction between NLRP3 and NEK7. Biochem Biophys Res Commun 517: 338-345, 2019.

33. Jiang Y, Du H, Liu X, Fu X, Li X and Cao Q: Artemisinin alleviates atherosclerotic lesion by reducing macrophage inflammation via regulation of $\mathrm{AMPK} / \mathrm{NF}-\varkappa \mathrm{B} / \mathrm{NLRP} 3$ inflammasomes pathway. J Drug Target 16: 1-10, 2019.

34. Lu BW, Baum L, So KF, Chiu K and Xie LK: More than anti-malarial agents: Therapeutic potential of artemisinins in neurodegeneration. Neural Regen Res 14: 1494-1498, 2019.

This work is licensed under a Creative Commons Attribution-NonCommercial-NoDerivatives 4.0 International (CC BY-NC-ND 4.0) License. 